\title{
UAV-Enabled 3D Indoor Positioning and Navigation Based on VLC
}

\author{
Guanchong Niu ${ }^{1,2}$, Junpeng Zhang ${ }^{1}$, Sheng Guo ${ }^{1}$, Man-On Pun ${ }^{1,2 \ddagger}$, Chung Shue Chen ${ }^{3}$ \\ ${ }^{1}$ School of Science and Engineering, The Chinese University of Hong Kong, Shenzhen, China \\ ${ }^{2}$ Shenzhen Research Institute of Big Data, Shenzhen, China \\ ${ }^{3}$ Nokia Bell Labs, Paris-Saclay, Route de Villejust, 91620 Nozay, France
}

\begin{abstract}
The 3D indoor positioning and indoor navigation (IPIN) system is of great significance for promoting and expanding indoor intelligent services and applications. The rapid development of unmanned aerial vehicles (UAVs) has provided new opportunities in this field. However, in contrast to their outdoor applications, IPIN for UAVs is more challenging since the Global Positioning System (GPS) is in general inaccessible in indoor environments. In this work, we propose a UAV-enabled 3D IPIN system based on visible light communication (VLC). Firstly, a novel VLC-based indoor positioning scheme is developed using a fusion algorithm based on the dynamic time warping (DTW) method with visible light intensity sequence (VLIS) and inertial measurement unit (IMU) data. To reduce the workload of fingerprint measurements, we propose to modularize a floor site using a standard symmetric structure for VLC positioning. In this manner, the navigation can be achieved by recognizing the edge of each module. Furthermore, since the sampling frequency of IMU is much higher than that of VLIS, discrete Kalman filter (KF) is introduced to correct the location measured by IMU when VLIS is unavailable. A proof-of-concept IPIN prototype is constructed. Field experiments confirm the effectiveness of our proposed IPIN system.
\end{abstract}

Index Terms-UAV, Indoor Positioning, Indoor Navigation, VLC, DTW.

\section{INTRODUCTION}

The vigorous development of new smart equipment has greatly promoted the rapid growth of indoor positioning and indoor navigation (IPIN) systems. Unmanned aerial vehicle (UAV) is a representative example. At present, the application scenarios of UAVs are mainly outdoors. However, UAVs can also be used in practical indoor environments such as hospitals, greenhouse, manufacturing factories, and nuclear power plants. Endowed with high mobility and low cost, UAVs have a wide range of applications, including indoor mapping and measurements [1]. However, considerable research efforts on IPIN systems are still required to materialize the benefits promised by UAVs in practice.

To address the challenges for global navigation satellite system (GNSS)-denied indoor scenarios, a few IPIN schemes have been proposed to break the limitation of traditional GNSS-based outdoor algorithms such as the shadow matching

This work was supported in part by Shenzhen Institute of Artificial Intelligence and Robotics for Society (AIRS) under Grant No. AC01202005001, the Shenzhen Science and Technology Innovation Committee under Grant No. ZDSYS20170725140921348 and the National Natural Science Foundation of China under Grant No. 61731018.

¥ Corresponding author, email: SimonPun@cuhk.edu.cn algorithm and the LiDAR digital elevation model (DEM)aided algorithm [2]. In conventional robotic studies, simultaneous localization and mapping (SLAM) technology based on a LiDAR or camera has been widely investigated for IPIN service for the GNSS-denied scenarios. However, the robustness and accuracy of vision-based methods cannot be guaranteed especially for the environment with repeated or similar patterns while the 3D LiDAR is usually too large and expensive for a UAV [3]. Alternatively, an IPIN algorithm based on the difference in horizontal and vertical 2D magnetic field intensity (MFI) measurements was proposed in [4]. However, field results suggested that the positioning accuracy is highly dependent on the quality of the magnetic field fingerprint. Unfortunately, the magnetic field fingerprint is often unstable, which makes the existing MFI-based method impractical. A 3D indoor positioning algorithm for UAVs was developed with spread spectrum ultrasound and Time-ofFlight (ToF) cameras in [5]. However, the interference caused by non-line-of-sight (NLoS) transmissions is noticeable.

The development of micro-electromechanical systems technology makes it possible that small low-cost inertial measurement unit (IMU) can be easily implemented on a UAV [6]. Despite that the IMU synchronous output of acceleration and altitude data can be used to provide precise 3D navigation during a short period, the IMU data may accumulate nonnegligible drift errors. To settle this problem, ultra-wideband (UWB)-based schemes provide an alternative solution. The UWB bandwidth enables high-precision ToF measurements in wireless communication. In [7], UWB and IMU data are fused to improve the positioning quality of UAV in takeoff and landing scenarios.

Recently, visible light communication (VLC)-based localization, built upon conventional light-emitting diodes (LEDs) and photodiodes (PDs) or optical cameras, has attracted much research interests due to its many advantages including its high positioning accuracy, license-free operation, no electromagnetic interference and low cost. In [8], an optical camera is utilized to estimate the distance between LEDs and the camera based on the relative positions of received pixels with respect to the size of captured photos. Similar to the classical triangulation algorithm [5], the distance estimation relied on the complicated VLC channel model. However, it is difficult to develop accurate VLC channel models as it requires accurate prior knowledge about the channel characteristics 


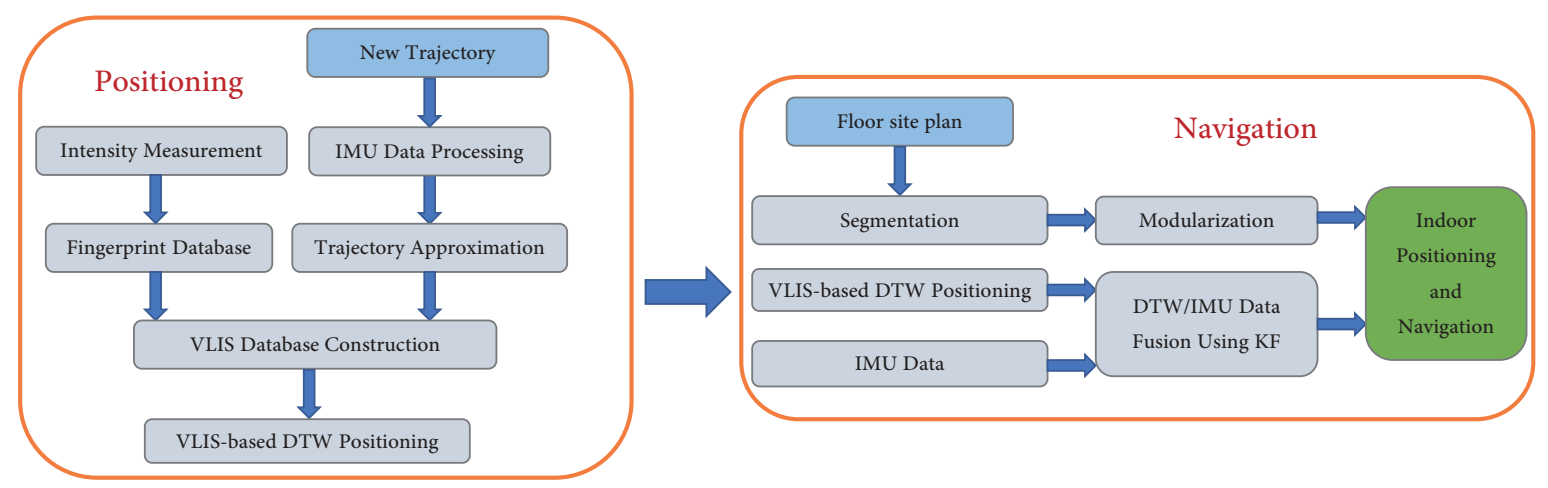

Fig. 1. Proposed VILS/IMU data fusion algorithm.

such as the irradiance and the incidence angle. To cope with this challenge, a fingerprint-based algorithm with machine learning (ML) techniques was developed in [9], where the channel model is not required. Despite the high accuracy, it is impractical to construct the fingerprint database for a large building due to the heavy workload.

In this work, we will develop a novel VLC-based 3D IPIN system for UAVs equipped with IMU and PDs as depicted in Fig. 1. The system is divided into two parts, namely positioning and navigation. By implementing the DTW algorithm, the $3 \mathrm{D}$ position can be estimated in a standard module. Then, the navigation can be realized using the modularized floor site. In addition, IMU can be utilized to measure the relative displacement while PDs can measure the intensity of light. The light intensity of each LED can be measured by a PD using the approaches such as time division multiple access (TDMA) [5]. The main contributions of our proposed new solution and system can be summarized as follows:

- We propose a low-cost IPIN system for UAVs using regular IMU and PDs that are commonly available on the market. In contrast to conventional database comprised of single fingerprinting, the proposed positioning system is robust to resist the stochastic noise by using a dynamic time warping (DTW) algorithm with visible light intensity sequence (VLIS).

- To reduce the workload for the construction of the fingerprint database, we propose to modularize the indoor scenarios using symmetric structure modules that are impossible for fingerprint algorithms based on UWB or magnetic field. After installing multiple modules in a floor site, the navigation can be achieved by detecting the edge of each module.

- Since the sampling frequency of IMU is usually much higher than that of VLIS measurement, Kalman filter (KF) can be utilized to predict the location in the absence of VLIS. By fusing IMU and VLIS, we can substantially reduce the positioning error as compared to the conventional DTW algorithm. Finally, extensive experiments have been conducted to prove the effectiveness of the proposed system.

\section{VLIS-BASEd INDOoR Positioning B Y THE Fusion OF VILS AND IMU DATA}

Light intensity collected at different locations can be used as the fingerprint database to determine the location of an object with PD. However, the intensity fingerprint collected from PD exhibits unstable intensities caused by stochastic drift even for different locations within a small area. To cope with the stochastic drift and realize robust indoor positioning, a fusion algorithm based on VLIS and IMU data is established in the sequel.

\section{A. Displacement Calculation by IMU}

We first build a suitable coordinate system to analyze data for IMU. The acceleration data collected from IMU can be transformed from the body coordinate system to a global coordinate system using the following matrix:

$$
\boldsymbol{R}=\left[\begin{array}{ccc}
c_{\gamma} c_{\psi}+s_{\gamma} s_{\psi} s_{\theta} & -c_{\gamma} s_{\psi}+s_{\gamma} c_{\psi} s_{\theta} & -s_{\gamma} c_{\theta} \\
s_{\psi} c_{\theta} & c_{\psi} c_{\theta} & s_{\theta} \\
s_{\gamma} c \psi+c_{\gamma} s_{\psi} s_{\theta} & -s \gamma s_{\psi}-c_{\gamma} c_{\psi} s_{\theta} & c_{\gamma} c_{\theta}
\end{array}\right]
$$

where $c_{\alpha}=\cos (\alpha)$ and $s_{\alpha}=\sin (\alpha)$ with $\gamma, \theta$ and $\phi$ being the roll, pitch and yaw, respectively.

The accelerations for the global coordinate system can be represented as

$$
\boldsymbol{a}_{g}=\boldsymbol{R} \boldsymbol{a}_{b}+\boldsymbol{G},
$$

where $\boldsymbol{a}_{b}$ is the acceleration in the body coordinate measured by the accelerometer and $\boldsymbol{G}=[0,0,-g]^{T}$ with $g$ being the gravity acceleration.

Subsequently, at the $t$-th time instant, the position after a displacement can be estimated by

$$
\boldsymbol{x}_{t}=\boldsymbol{x}_{t-1}+\sum_{k=0}^{K}\left[v_{k} \Delta T+\frac{1}{2} \boldsymbol{a}_{g}^{(k)} \Delta T^{2}\right]
$$

where $k=0,1, \cdots, K$ is the sample index of the IMU data with a sampling interval period of $\Delta T$ and $v_{k}$ is the velocity at the $k$-th instant. In $2 \mathrm{D} / 3 \mathrm{D}$ case, $\boldsymbol{x}_{t}$ is a $2 \mathrm{D} / 3 \mathrm{D}$ coordinate of the object at the $t$-th instant. 


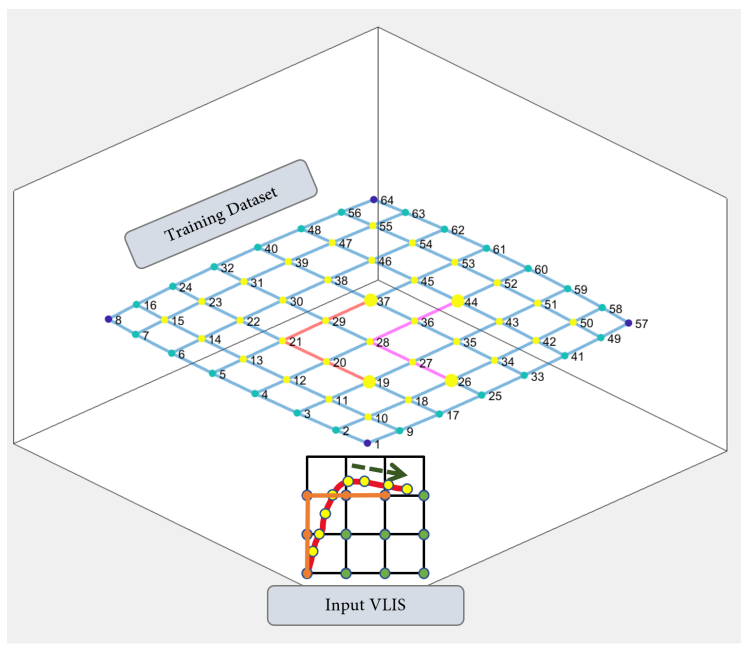

Fig. 2. Trajectory approximation and training data generation.

\section{B. 2D Case Positioning Based on DTW Matching}

For the 2D positioning case, we can build a fingerprint database by dividing the space as squares of size $\lambda \times \lambda$ as shown in Fig. 2. To construct the fingerprint database for each position, PD is utilized to measure the intensity of each LED in TDMA transmission.

1) Input Trajectory: For an arbitrary trajectory, we can use the IMU data to generate a VLIS while the complexity can be also reduced by shortening the length of the sequence. Inspection of (3) suggests that the relative displacement in a short time can be calculated with good accuracy in a straightforward manner. We observe a short time interval of $T$ in which the trajectory is divided into $N$ sampling positions with a sequence set $\boldsymbol{X}=\left[\boldsymbol{x}_{0}, \cdots, \boldsymbol{x}_{n}, \cdots, \boldsymbol{x}_{N}\right]$. In the beginning of each trajectory, we assume $\boldsymbol{x}_{0}=[0,0]^{T}$ for the $2 \mathrm{D}$ case to calculate the relative displacement as shown in Fig. 2. Since the previous position is not required for the input trajectory approximation, the accumulated error of IMU can be suppressed.

2) Training Dataset: For the DTW algorithm, a fingerprint sequence is required in advance. As shown in Fig. 2, we firstly build a square for the given trajectory. The position sequence can be rewritten as $\boldsymbol{X}=\left[\boldsymbol{v}_{1}^{T}, \boldsymbol{v}_{2}^{T}\right]$, where $\boldsymbol{v}_{1}^{T}$ and $\boldsymbol{v}_{2}^{T}$ are the position vectors in $x$ and $y$ axis, respectively. The range of trajectory is limited by a small space of width $W=\left\|\boldsymbol{v}_{1}^{T}\right\|_{\infty}$ and length $L=\left\|\boldsymbol{v}_{2}^{T}\right\|_{\infty}$, respectively. The number of required fingerprint samples is $N_{W} \times N_{L}$, where

$$
N_{W}=\lceil W / \lambda\rceil, \quad N_{L}=\lceil W / \lambda\rceil,
$$

with $\lceil\cdot\rceil$ being the largest integer not greater than the quantity enclosed.

Then, the fingerprint VLIS set can be represented as

$$
\boldsymbol{I}=\left[\begin{array}{cccc}
I_{00} & I_{01} & \cdots & I_{0 N_{L}} \\
\vdots & \vdots & \ddots & \vdots \\
I_{N_{W} 0} & I_{N_{W} 1} & \cdots & I_{N_{W} N_{L}}
\end{array}\right]
$$

with a corresponding position set

$$
\boldsymbol{P}=\left[\begin{array}{cccc}
\boldsymbol{p}_{00} & \boldsymbol{p}_{01} & \cdots & \boldsymbol{p}_{0 N_{L}} \\
\vdots & \vdots & \ddots & \vdots \\
\boldsymbol{p}_{N_{W} 0} & \boldsymbol{p}_{N_{W} 1} & \cdots & \boldsymbol{p}_{N_{W} N_{L}}
\end{array}\right]
$$

Thus, the trajectory can be approximated as a sequence in the fingerprint database:

$$
\boldsymbol{x}_{n}^{*}=\underset{\substack{i=0,1, \cdots, N_{W}, j=0,1, \cdots, N_{L}}}{\arg \min }\left\|\boldsymbol{p}_{i j}-\boldsymbol{x}_{n}\right\|_{2}, \quad \text { for } \quad n=0,1, \cdots N .
$$

As shown in Fig. 2, the input approximated VLIS $\boldsymbol{I}^{*}$ is the intensity sequence corresponding to the position sequence $\boldsymbol{X}^{*}=\left[\boldsymbol{x}_{0}^{*}, \boldsymbol{x}_{1}^{*}, \cdots, \boldsymbol{x}_{N}^{*}\right]$. Subsequently, the training database $\mathcal{T}$ is constructed based on the structure of $\boldsymbol{X}^{*}$. For instance, the red and pink trajectory sequences depicted in Fig. 2, i.e., the sequence with index $[26,27,28,36,44]$ and $[19,20,21,29,37]$, are the two VLIS sets in $\mathcal{T}$. In this way, a pre-trained fingerprint database $\mathcal{T}$ can be constructed directly. By calculating the DTW scores for $\boldsymbol{X}^{*}$ and $\mathcal{T}$, the similarity score can be derived before the position is determined by the sequence with the smallest score.

\section{VLC-BASED INDOOR NAVIGATION}

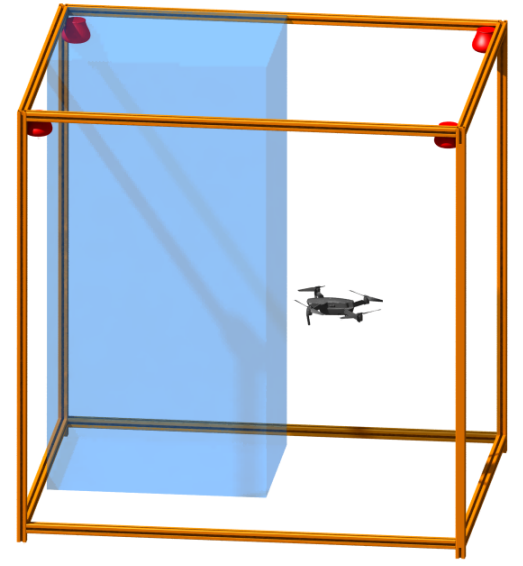

Fig. 3. Fingerprint measurement for a 3D module.

In fingerprint-based algorithms, the main drawback is that fingerprints are required to be collected over the deployment site. Furthermore, extension data collection is required to achieve higher positioning accuracy. Such data collection workload is usually unaffordable in practice. In contrast to the conventional UWB- or magnetic-based algorithms, VLIS provides a possible method for modularization since the light intensity can be controlled and the deployment of LEDs can be deployed manually. Motivated by this observation, we propose to first classify the floor site into several types of modules. In this way, we only need to measure the fingerprints of predefined modules such that the workload can be significantly reduced. 


\section{A. Modularization}

In this work, a standard symmetric module is built to further reduce the complexity of fingerprint measurements as shown in Fig. 3, where a simple example is developed. By taking advantage of the symmetric property of four LEDs, we only need to measure the fingerprint data in the blue space. Using data processing, the fingerprints of other parts can be directly obtained without further measurement.

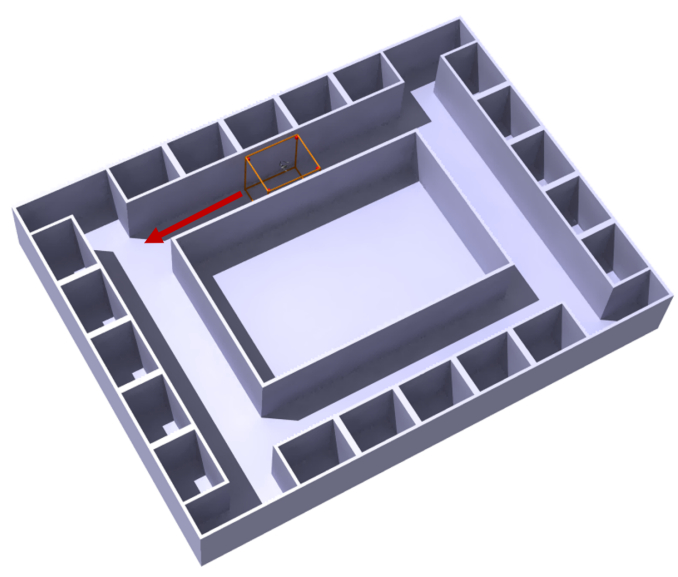

Fig. 4. Navigation scheme in a floor site.

As shown in Fig. 4, the floor site can be directly modularized by the predefined module by taking advantage of the similarity in a floor site. Thus, we can localize the position at a floor site by detecting the edge of each module after modularizing the floor site.

The above model can be applied to significantly reduce complexity and improve accuracy. Specifically, the key node for navigation can be selected as the edge of each module. Once the measured VLIS is detected across the edge, the object moves from one module to another one. In this manner, the navigation of an object can be developed.

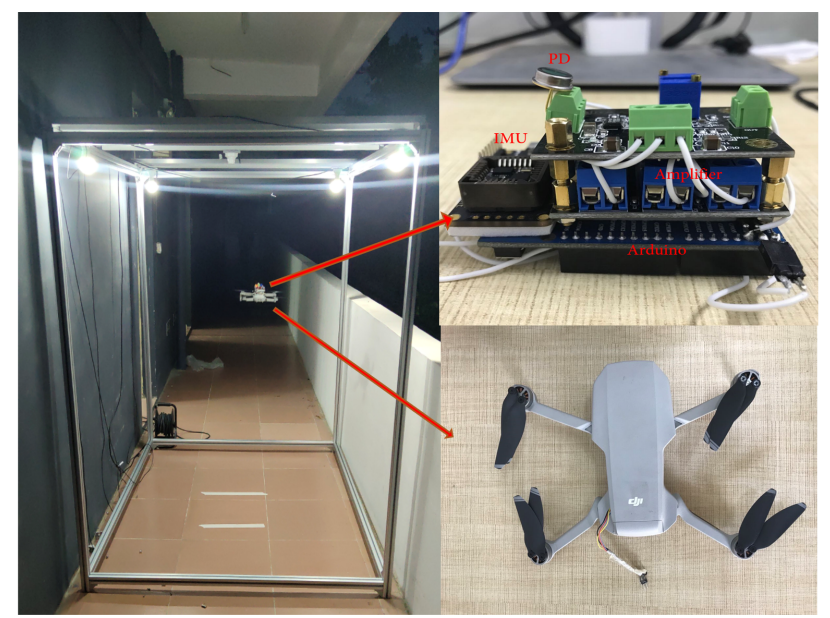

Fig. 5. UAV used in this experiment.

\section{B. UAV-Enabled 3D IPIN System}

In this paper, we propose a UAV-enabled 3D IPIN system utilizing VLIS and IMU data as depicted in Fig. 5. Due to the characteristics of quadrotor UAVs, the body of a UAV is always horizontal to the ground at a static state. Thus, the PD equipped on the UAV is vertical to the ground. Furthermore, despite that IMU is usually a standard component in a UAV, it is difficult to read the IMU data. As a result, we have to add an additional IMU on UAV. In addition, PD is not typically available on UAV. Thus, PD has to be added. To conduct the experiment, we only control the UAV to realize 3D movement while an Arduino connected with a computer is utilized to collect and transmit the data measured from IMU and PD.

It is clear that the accuracy of positioning and navigation is highly relevant to the resolution of the fingerprint database. If the space between the two fingerprints is $\lambda$, the average resolution should be $\lambda / 2$. However, there will be much more workload needed for higher resolution in practice. On the other hand, the sampling frequency of VLIS is much lower than that of IMU. To reduce the accumulated error of IMUonly period, $\mathrm{KF}$ is introduced to fuse data from IMU and PD for the proposed IPIN systems in the next section.

\section{VLIS/IMU DATA FUSION FOR IPIN SYSTEMS USING DiSCRETE KALMAN FILTER}

Despite that DTW can provide good performance, its update frequency is limited by the time required for VLIS measurement. In contrast, IMU data can be updated much frequently while suffering from accumulated errors. To cope with these challenges, we propose a data fusion algorithm using VLIS and IMU data based on the discrete KF in the following.

\section{A. Discrete Kalman Filter}

The KF is proposed for dynamic systems to perform state estimation by exploiting sensor measurements. A typical KF can be described using the following discrete-time state space model:

$$
\begin{aligned}
\boldsymbol{s}_{t+1} & =\boldsymbol{A} \boldsymbol{s}_{t}+\boldsymbol{B} \boldsymbol{R}_{t} \boldsymbol{u}_{t}+\boldsymbol{\beta}, \\
\boldsymbol{p}_{t} & =\boldsymbol{C} \boldsymbol{s}_{t}+\boldsymbol{\omega}
\end{aligned}
$$

where $\boldsymbol{s}_{t}=\left[x_{t}, y_{t}, z_{t}, v_{x, t}, v_{y, t}, v_{z, t}\right]^{T}$ is the state vector. At time instant $t$, the input parameter $\boldsymbol{u}_{t}=\left[a_{x, t}, a_{y, t}, a_{z, t}\right]^{T}$ is the acceleration at $x, y$ and $z$ axis of the global coordinate system, respectively, while the output result $\boldsymbol{p}_{t}=\left[x_{t}, y_{t}, z_{t}\right]^{T}$ represents the 3D aerial position coordinates of an object in a global Cartesian coordinate system. The three dimension rotation matrix $\boldsymbol{R}_{t}$ is $\boldsymbol{R}$ represented by (1) at the $t$-th time instant to estimate the user's propagated position in the global coordinate. Finally, $\boldsymbol{\beta}$ and $\boldsymbol{\omega}$ are the Gaussian noise with zero mean separately.

The transition matrix $\boldsymbol{A} \in \mathbb{R}^{6 \times 6}$, control matrix $\boldsymbol{B} \in \mathbb{R}^{6 \times 3}$, and the output matrix $C \in \mathbb{R}^{3 \times 6}$ can be represented as

$$
\boldsymbol{A}=\left[\begin{array}{cc}
\boldsymbol{I}_{3} & \Delta T \boldsymbol{I}_{3} \\
\mathbf{0}_{3} & \boldsymbol{I}_{3}
\end{array}\right], \boldsymbol{B}=\left[\begin{array}{c}
\frac{1}{2} \Delta T^{2} \boldsymbol{I}_{3} \\
\Delta T \boldsymbol{I}_{3}
\end{array}\right], \boldsymbol{C}=\left[\begin{array}{l}
\boldsymbol{I}_{3} \\
\mathbf{0}_{3}
\end{array}\right]^{T},
$$


where $\boldsymbol{I}_{3} \in \mathbb{R}^{3 \times 3}$ is an identity matrix and $\mathbf{0}_{3} \in \mathbb{R}^{3 \times 3}$ is a zero matrix.

\section{B. VLIS/IMU Data Fusion Using Discrete Kalman Filter}

As the VLIS is utilized to infer the position of the UAV, we can get the position estimated by the DTW algorithm once a VLIS is measured at instant $t$. The trajectory corresponding to the end of trajectory with matched VLIS is considered as the most reliable position denoted by $\boldsymbol{p}_{t}$. Since the sampling frequency of IMU is much larger than the fingerprint matching, IMU can be used to estimate the position more frequently. As a result, we can use the KF to realize a better accuracy.

Correspondingly, the state space model of IMU for KF in (8) can be used to derive the VLIS/IMU fusion algorithm. The detailed formulation of position estimation based on KF is listed as follows:

$$
\begin{aligned}
\hat{\boldsymbol{s}}_{t \mid t-1} & =\boldsymbol{A} \hat{\boldsymbol{s}}_{t-1 \mid t-1}, \\
\boldsymbol{\Sigma}_{t \mid t-1} & =\boldsymbol{A} \boldsymbol{\Sigma}_{t-1 \mid t-1} \boldsymbol{A}^{T}+\boldsymbol{Q}, \\
\boldsymbol{e}_{t} & =\boldsymbol{p}_{t}-\boldsymbol{C} \hat{\boldsymbol{s}}_{t \mid t-1}, \\
\boldsymbol{E}_{t} & =\boldsymbol{C} \boldsymbol{\Sigma}_{t \mid t-1} \boldsymbol{C}^{T}+\sigma^{2} \boldsymbol{I}_{M}, \\
\boldsymbol{K}_{t} & =\boldsymbol{\Sigma}_{t \mid t-1} \boldsymbol{C}^{T} \boldsymbol{E}_{t}^{-1} \\
\hat{\boldsymbol{s}}_{t \mid t} & =\hat{\boldsymbol{s}}_{t \mid t-1}+\boldsymbol{K}_{t} e_{t}, \\
\boldsymbol{\Sigma}_{t \mid t} & =\boldsymbol{\Sigma}_{t \mid t-1}-\boldsymbol{K}_{t} \boldsymbol{C} \boldsymbol{\Sigma}_{t \mid t-1}, \\
\hat{\boldsymbol{p}}_{t \mid t} & =\boldsymbol{C} \hat{\boldsymbol{s}}_{t \mid t} \\
\boldsymbol{\Sigma}_{t \mid t}^{p} & =\boldsymbol{C} \boldsymbol{\Sigma}_{t \mid t} \boldsymbol{C}^{T}
\end{aligned}
$$

where $\boldsymbol{Q}=\mathbb{E}\left[\boldsymbol{\omega} \boldsymbol{\omega}^{T}\right]$ and $\sigma^{2}$ is the variance of Gaussian noise $\boldsymbol{\beta}$. $\hat{\boldsymbol{s}}_{t \mid t-1}$ is the prior estimate of the state vector with corresponding covariance matrix $\boldsymbol{\Sigma}_{t \mid t-1}$. The measurement residual is denoted by $\boldsymbol{e}_{t}$ with covariance matrix $\boldsymbol{E}_{t} . \boldsymbol{K}_{t}$ is the Kalman gain and $\hat{\boldsymbol{p}}_{t \mid t}$ is the posterior estimation of output with covariance $\Sigma_{t \mid t}^{p}$. We assume that the initial position and speed of a UAV is given as $\boldsymbol{p}_{0}=\left[x_{0}, y_{0}, z_{0}\right]^{T}$ and $\boldsymbol{v}_{0}=\left[v_{x, 0}, v_{y, 0}, v_{z, 0}\right]^{T}$. Therefore, the initial state vector is $\boldsymbol{s}_{0}=\left[\boldsymbol{p}_{0}^{T}, \boldsymbol{v}_{0}^{T}\right]^{T}$ with covariance $\boldsymbol{\Sigma}_{0}$.

The above KF procedure works iteratively and combines the data from the two sensors. At each iteration, it first uses the data from IMU to predict the position and the velocity of the object before VLIS is obtained. Once the position is calculated by the DTW algorithm, it will update the position using Kalman gain as a weighted average. Thereby the updated position will be closer to the estimated position with higher certainty. Since the sampling frequency of IMU is much larger than DTW, the trajectory can be corrected by KF during the period lacking VLIS.

\section{EXPERIMENTAL RESULTS}

To evaluate the performance of the proposed IPIN system, a proof-of-concept experiment is conducted below. A UAV equipped with PD, IMU and Arduino is depicted in Fig. 5. We use wireless-enabled Arduino D1 which can receive the amplified signal measured from a PD (OSD15-E). The components used in this work are detailed in Table I.
TABLE I

COMPONENT DESCRIPTION.

\begin{tabular}{l|l|l}
\hline Components & Type & Function \\
\hline Photodiode (PD) & OSD15-E & Measure the light intensity \\
\hline Amplifier & IV Amplifier & Amplify the current of PD \\
\hline Arduino & WeMos D1 R1 & Wireless control \\
\hline IMU & MPU 9250 & $\begin{array}{l}\text { Calculate the relative dis- } \\
\text { placement of distance and } \\
\text { direction }\end{array}$ \\
\hline UAV & DJI Mavic Air & Realize 3D movement \\
\hline LED & Panasonic 25W & Transmit light \\
\hline
\end{tabular}

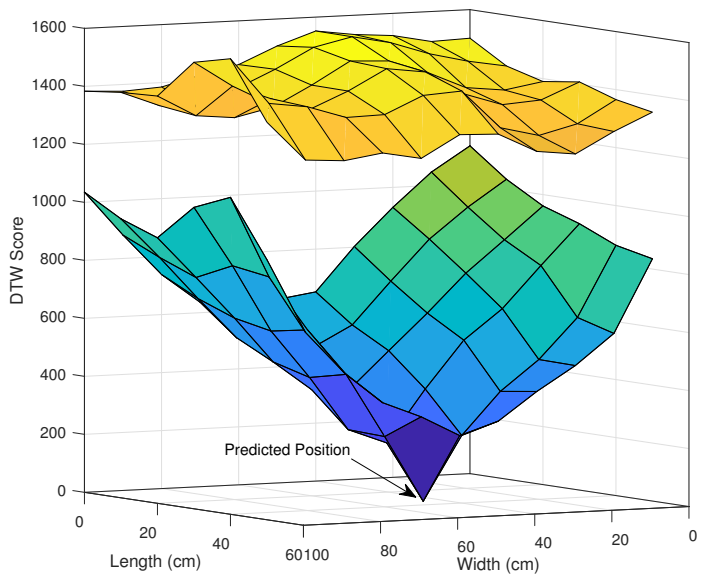

Fig. 6. 3D positioning based on DTW algorithm

For the offline training, the fingerprints are measured with an interval $0.1 \times 0.1 \times 0.1 \mathrm{~m}^{3}$ for four LEDs. By using the approximated trajectory described in Section II, we can obtain the location by comparing the similarity of VLIS using the DTW algorithm.

As shown in Fig. 6, a trajectory with VLIS containing five fingerprints is localized by utilizing the DTW algorithm. To avoid confusion, the given trajectory moves in a $2 \mathrm{D}$ horizontal plane while we compare the fingerprints with its adjacent layer. A layer means the fingerprint database measured at a specific height. Thereby the position is predicted in the sequence with the smallest DTW score.

In Fig. 7, the time cost of the proposed DTW algorithm is investigated. It is clear that the time cost will increase with the increment of space. In practice, the time cost is less than $0.5 \mathrm{~s}$, which shows a promising method for the real-time positioning system.

Next, we show the effectiveness of the proposed KF fusion algorithm using the VLIS and IMU data. The comparison of different schemes for trajectory navigation is shown in Fig. 8. The trajectory corrected by KF is closer to the real trajectory. In contrast, the trajectory using IMU accumulates errors for a long distance while the DTW scheme has random drifts around the ground truth.

Finally, the average error of 10 trajectories for different 


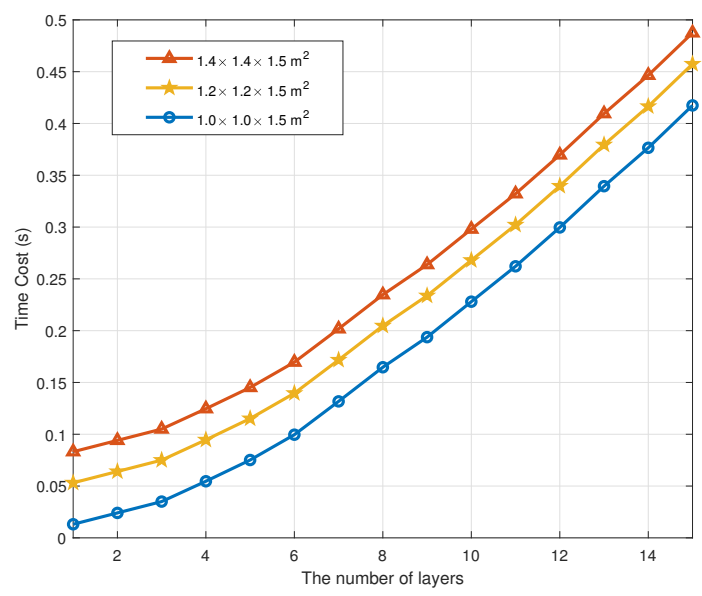

Fig. 7. Time cost comparison for different sizes of space.

schemes is evaluated in Fig. 9. Although the error of IMU scheme is very small in the beginning, it increases quickly as compared to the DTW and KF schemes. The average error of $\mathrm{KF}$ scheme converges to about $4 \mathrm{~cm}$. As shown in this result, the KF scheme has a higher sampling frequency and the accuracy is improved as well.

\section{CONCLUSION}

In this paper, we have established a UAV-enabled IPIN system using VLIS/IMU data fusion. Firstly, a DTW-based indoor positioning algorithm has been developed by approximating the trajectory with pre-measured fingerprint sequences. Next, we have proposed to modularize the floor site into a symmetric structure with four LEDs to cope with the main challenge of heavy workload for fingerprint measurements. Furthermore, we have proposed to employ KF to reduce the stochastic errors caused by VILS and IMU. Finally, experiments have been conducted to prove the validity of the proposed IPIN system.

\section{ACKNOWLEDGMENT}

We would like to thank Dr. Siu-Wai Ho (University of Adelaide) and Dr. Fabio Pianese (Nokia Bell Lab) for some insightful discussions and comments.

\section{REFERENCES}

[1] Q. Zhang, G. Niu, and M. Pun, "Large-area super-resolution 3D digital maps for indoor and outdoor wireless channel modeling," in 2018 IEEE 87th Vehicular Technology Conference (VTC Spring), June 2018, pp. 1-6.

[2] R. Yozevitch and B. B. Moshe, "A robust shadow matching algorithm for GNSS positioning," Navigation: Journal of The Institute of Navigation, vol. 62, no. 2, pp. 95-109, 2015.

[3] T. Caselitz, B. Steder, M. Ruhnke, and W. Burgard, "Monocular camera localization in 3D Lidar maps," in 2016 IEEE/RSJ International Conference on Intelligent Robots and Systems (IROS). IEEE, 2016, pp. 1926-1931.

[4] B. Kim and S.-H. Kong, "A novel indoor positioning technique using magnetic fingerprint difference," IEEE Transactions on Instrumentation and Measurement, vol. 65, no. 9, pp. 2035-2045, 2016.

[5] M. Yasir, S. Ho, and B. N. Vellambi, "Indoor positioning system using visible light and accelerometer," Journal of Lightwave Technology, vol. 32, no. 19, pp. 3306-3316, 2014.

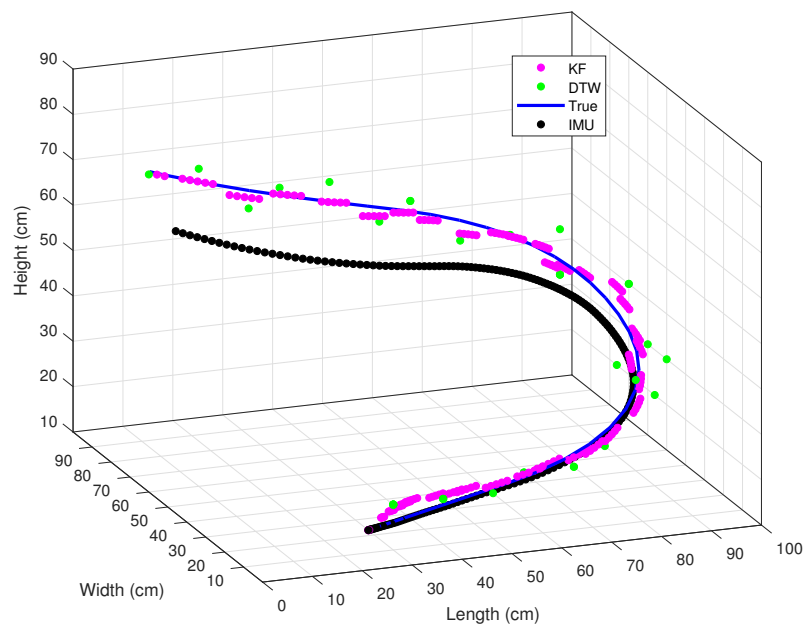

Fig. 8. Correction of IMU Trajectory using KF for indoor navigation.

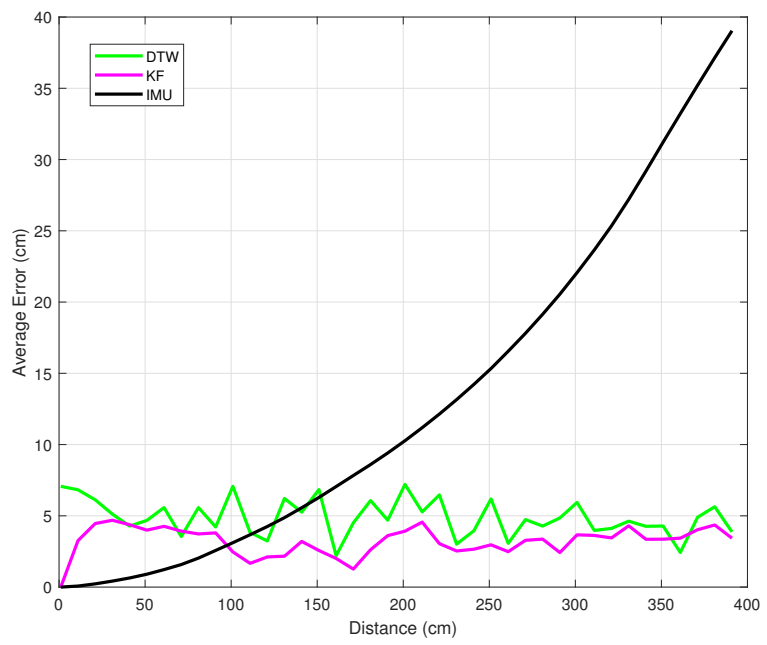

Fig. 9. Average error comparison.

[6] C. Eling, L. Klingbeil, and H. Kuhlmann, "Real-time single-frequency GPS/MEMS-IMU attitude determination of lightweight UAVs," Sensors, vol. 15 , no. 10, pp. 26212-26235, 2015.

[7] K. Hausman, S. Weiss, R. Brockers, L. Matthies, and G. S. Sukhatme, "Self-calibrating multi-sensor fusion with probabilistic measurement validation for seamless sensor switching on a UAV," in 2016 IEEE International Conference on Robotics and Automation (ICRA). IEEE, 2016, pp. 4289-4296.

[8] B. Lin, Z. Ghassemlooy, C. Lin, X. Tang, Y. Li, and S. Zhang, "An indoor visible light positioning system based on optical camera communications," IEEE Photonics Technology Letters, vol. 29, no. 7, pp. 579-582, 2017.

[9] F. Alam, M. T. Chew, T. Wenge, and G. S. Gupta, "An accurate visible light positioning system using regenerated fingerprint database based on calibrated propagation model," IEEE Transactions on Instrumentation and Measurement, vol. 68, no. 8, pp. 2714-2723, 2018. 